\title{
INMERSIÓN DE LOS HOTELES BOUTIQUE EN EL GRAN GUAYAQUIL, ANÁLISIS DE LA OFERTA Y PERCEPCIÓN DEL SERVICIO: CASO HOTEL DEL PARQUE IMMERSION OF THE BOUTIQUE HOTELS IN THE GRAN GUAYAQUIL, ANALYSIS OF THE OFFER AND PERCEPTION OF THE SERVICE: CASE OF HOTEL DEL PARQUE
}

\author{
Alejandra Ycaza Calderón, Mgtr. \\ Universidad Tecnológica ECOTEC, Samborondón, Ecuador. \\ mycaza@ecotec.edu.ec \\ Guillermo Granja Cañizares, Mgtr. \\ iD https://orcid.org/0000-0002-7619-3350 \\ Universidad Tecnológica ECOTEC, Samborondón, Ecuador. \\ ggranja@ecotec.edu.ec
}

\section{ARTÍCULO DE INVESTIGACIÓN}

Recibido: 18 de junio de 2021

Aceptado: 4 de agosto de 2021

\section{RESUMEN}

El presente artículo tiene como objetivo analizar la importancia del servicio y oferta en los hoteles boutique para mejorar su posicionamiento y rentabilidad en un mercado creciente, en este caso particular en el hotel del Parque en las afueras de la ciudad de Guayaquil. Para este análisis se consideró la encuesta para observar primeramente el posicionamiento del hotel frente a su competencia cantonal y nacional, la misma que fue realizada a un grupo de 300 personas de público general, externo y aleatorio; además se realizó un estudio de campo en el hotel, y se analizaron los comentarios de huéspedes de tres de las principales OTAS (Agencias de viaje virtual) donde los clientes compartieron su percepción sobre el servicio y la oferta recibida. También se analizó la oferta de paquetes y servicios con los que trabaja actualmente el hotel. Los resultados demostraron el grado de posicionamiento del hotel dentro del sector y en el mercado de los hoteles de lujo y boutique desde una perspectiva general, así como la percepción de satisfacción del huésped durante su estadía, donde se adicionaron los puntos claves que hicieron que su experiencia cumpla su ciclo de servicio de manera exitosa. La reflexión final indica que en un hotel boutique el servicio superior y de calidad, así como la capacidad del hotel para adelantarse a las necesidades del huésped y sorprenderlo de manera creativa y elegante, conllevan a la creación de experiencias que serán memorables y únicas, y que contribuirán al crecimiento de la marca y a la rentabilidad.

Palabras claves: hotel boutique, estrategias de venta, oferta, servicio al cliente, hoteles de lujo 


\section{ABSTRACT}

This article aims to analyze the importance of the service and offer in boutique hotels to improve their positioning and profitability in a growing market, in this particular case in the Park hotel on the outskirts of the city of Guayaquil. For this analysis, the survey was considered to first observe the position of the hotel in relation to its cantonal and national competition, which was carried out to a group of 300 people from the general, external and random public; In addition, a field study was carried out in the hotel, and the comments of guests from three of the main OTAS (Virtual Travel Agencies) were analyzed, where customers shared their perception of the service and the offer received. The offer of packages and services with which the hotel is currently working was also analyzed. The results demonstrated the degree of positioning of the hotel within the sector and in the market of luxury and boutique hotels from a general perspective, as well as the perception of guest satisfaction during their stay, where the key points that made their stay were added. experience to complete your duty cycle successfully. The final reflection indicates that in a boutique hotel the superior and quality service, as well as the ability of the hotel to anticipate the needs of the guest and surprise him in a creative and elegant way, lead to the creation of experiences that will be memorable and unique, and that will contribute to brand growth and profitability.

Keywords: boutique hotel, sales strategies, offer, guest service, luxury hotel.

\section{INTRODUCCIÓN}

Los hoteles boutique hoy en día representan una oferta única y exclusiva dentro del mundo hotelero. Este tipo de hotel se caracteriza por ofrecer un alojamiento de lujo con un servicio que supera las expectativas de sus huéspedes, quienes están dispuestos a pagar una tarifa mucho más alta por percibir una experiencia inolvidable Revista Empresarial \& Laboral (2018).

La capacidad para sorprender a sus huéspedes, y de crear momentos incomparables a través del servicio ofrecido por el equipo humano, son claves para cumplir el ciclo de la excelencia que se transforma en ganancias y en el crecimiento de su marca.

Como lo indica Ycaza (2019, página 949) "si se logran comprender las necesidades del consumidor, se podrán desarrollar productos y servicios que proporcionen un valor superior para el cliente; y así fijar un precio (o tarifa en el caso de hotelería) logrando distribuir y comunicar de manera eficaz la oferta hacia el cliente final. De esta manera, la parte comercial podrá vender con mayor facilidad el producto que llegará al cliente correcto, en el momento correcto, por el canal correcto y al precio correcto". 
El Hotel del Parque, actualmente es el primer hotel de lujo de tipo boutique en el área denominada el Gran Guayaquil. Adicional a sus cómodas habitaciones y al servicio de hospedaje, encierra mucha historia por encontrarse en un edificio ícono de la arquitectura colonial de Guayaquil del siglo XIX, lo que lo convierte en parte del patrimonio histórico de la ciudad. El hotel se encuentra ubicado en la vía a Samborondón, dentro del Parque Histórico, atractivo turístico de gran importancia para Guayaquil, por su aporte a la cultura y al turismo. Empresa Pública Municipal de Turismo, Promoción Cívica y Relaciones Internacionales de Guayaquil, EP (2019).

El Hotel es parte de la cadena de hoteles "Oro Verde Hotels", cadena ecuatoriana con 7 hoteles de lujo alrededor del Ecuador: Oro Verde Cuenca, Oro Verde Machala, Oro Verde Manta, Villa Escalesia en Galápagos, Unipark, Oro Verde Guayaquil, y el Hotel del Parque en la vía a Samborondón, área considerada como parte del Gran Guayaquil. Es un edificio patrimonio de la ciudad, restaurado para convertirse en un establecimiento de hospedaje. Hotel del Parque Histórico (2020).

“Antiguamente fue conocido como el Hospicio Corazón de Jesús. La propiedad fue construida en 1891 por la institución de caridad más antigua de Guayaquil. Sería el primer hospicio en la ciudad, y sus fundadores, Manuel Galecio y su esposa, la señora Jesús Pereira, declararon que sólo debía dar refugio a hombres y mujeres sin hogar, ancianos o discapacitados". (Hotel del Parque Histórico Guayaquil, 2019)

Desde su apertura hasta la actualidad, el hotel se ha posicionado como uno de los más lujosos y con mejor servicio y gastronomía del sector. Recibe en sus instalaciones a personalidades diplomáticas y artísticas del más alto nivel, y por sus reconocimientos a nivel nacional e internacional, es un ejemplo importante para un estudio de caso basado principalmente en la percepción del cliente sobre su oferta y su servicio Booking Reseñas y Valoraciones (2020)

El objetivo general es determinar cómo se percibe el servicio en el Hotel del Parque dentro de la oferta de hoteles de lujo en Guayaquil.

\section{MARCO TEÓRICO}

Se parte de una fundamentación teórica de la literatura científica respecto a la experiencia, el posicionamiento, la identidad e imagen corporativa, para luego proceder al análisis de la oferta y la medición la percepción del servicio en el Hotel del Parque.

Es importante esto para poder comprender luego desde la perspectiva científica los resultados del diagnóstico y enriquecer luego las conclusiones alrededor del objeto de estudio. 
El estudio de la experiencia es una parte importante dentro de la empresa de servicios, ha sido tema de investigación en turismo desde la década de los 90's, desde proponer matrices para evaluar y gestionar la dimensión de las experiencias Beeho \& Prentice, (1997), hasta los argumentos que afirman la importancia de la economía de la experiencia que está basada principalmente en la calidad del servicio, según los estudios de Pine II \& Gilmore (1998) explica las características de las experiencias desde dos dimensiones: la participación del cliente y la conexión o relación con el entorno al momento de percibir un servicio o participar de una actividad, involucrando los 5 sentidos y cubriendo siempre una necesidad Sánchez Aguirre, Maldonado Alcudia, Martínez Vázquez, \& Salinas Villareal, (2017).

Como lo describe lbáñez \& Manzano (2007), uno de los puntos más importantes a destacar para determinar el valor de una marca, es su posicionamiento en el mercado. Este se define como "el acto de diseñar la oferta e imagen de una empresa con el fin de ocupar una posición diferente en la mente del consumidor". Es una de las estrategias más importantes del marketing ya que permiten esa diferenciación del producto o servicio en el mercado que crea la conexión con su cliente, aporta valor al consumidor e induce a la compra

Complementando lo anterior tener. Cisneros Garrido (2015) indica que, para obtener un adecuado posicionamiento, la estrategia debe considerarse desde su creación para ser sostenible y tener un punto diferenciador, no necesariamente hay que ser pioneros en la marca para lograr un posicionamiento exitoso, pero si debe ser percibida con el nivel de satisfacción del cliente. Adicionalmente, hay que tener en cuenta que la experiencia que tenga el cliente va a condicionar la estrategia del posicionamiento del producto o servicio sensiblemente. Es por esto, que debe adaptarse a los cambios en los niveles de experiencias que cada individuo vaya a

Los autores Rey, Zambrano, \& Zambrano (2015) concuerdan con la importancia de generar experiencias, y cómo la identidad e imagen corporativa a través de sus características más reconocibles, su prestigio, sus valores y su consideración social crean a su vez una reputación para la empresa. Con esto, la empresa se posiciona en la mente de sus consumidores, tanto con aspectos tangibles como intangibles.

Cuando se analiza el posicionamiento de una marca ya sea en un producto o un servicio, generalmente se identifican primero los comportamientos de compra y de consumo, para estudiar la identidad e imagen corporativa propuesta. Díaz-Bustamante (2013).

Como lo indican Kotler \& Bowen T. (2015): "El mercado es el conjunto de compradores reales y potenciales de un producto. Estos compradores comparten una necesidad o deseo particular que se puede satisfacer mediante relaciones de intercambio". 
Es por esto por lo que un hotel, y en especial un hotel boutique, debe conocer muy bien cuál es su mercado y entender sus necesidades para poder crear una oferta que conlleve a una experiencia final exitosa.

Hay que reconocer que, para el sector turístico, las emociones y la percepción del servicio son puntos claves que miden el valor con la calidad percibida por el turista. Este valor depende del sentido que tenga el producto o el servicio ofrecido. $Y$ cuando se ha logrado este objetivo se gana la credibilidad del consumidor en la existencia de valores compartidos entre la empresa y el cliente. Acosta, Fernandez, \& Mollón (2002)

De acuerdo a un artículo de Hosteltur edición LATAM sobre el informe para clasificación de hoteles realizado por la OMT en colaboración con una institución noruega (UNWTO, 2015), el documento identifica los criterios comunes entre los hoteles de 4 y 5 estrellas, proporcionando una valiosa información para aquellos destinos que desean revisar sus sistemas de clasificación de hoteles o establecer uno nuevo.

Entre los Hallazgos y observaciones claves sobre sistemas de clasificación hotelera se ha encontrado que ayuda a la administración de destinos y guía de mejor manera al consumidor, permite que se eleve la calidad del alojamiento sin importar el tamaño o si es de marca o independiente, generando un mejor posicionamiento en los hoteles pequeños Hoseltur LATAM (2015).

En el sector de alojamiento, los turistas han ido evolucionando en cuanto a preferencias y formas de llegar a lo que les gusta, han pasado de ser pasivos a ser participativos porque demandan la excelencia en el servicio y experiencias de calidad Morgan, Elbe, \& Curiel, (2019). Es por esto, que los establecimientos de hospedaje también han tenido que ir modificando su oferta, siendo innovadores y entendiendo que el turista ya no va a escoger un hotel solo porque necesita pernoctar en un lugar, sino porque le brindará algo adicional. Y así es como se han ido desarrollando los hoteles originales, con elegancia en su decoración, un toque de historia, cultura y arte, y por supuesto con un servicio de alta calidad, a este tipo de alojamiento se lo conoce como hotel boutique.

El término boutique de origen francés es definido por La Real Academia Española (RAE), como "una tienda de indumentaria de moda o de artículos selectos". Y es bajo esta misma premisa, que el término Hotel Boutique describe a un establecimiento selecto, único y lujoso que ofrece un servicio personalizado. La idea final es que la persona que se hospeda en uno de estos hoteles no tenga la misma experiencia en ninguna otra parte del mundo.

Como lo expresan Contardo \& Wenger (2012), los hoteles boutique cumplen el objetivo de poseer un elemento diferenciador, ya que son cálidos, confortables y de agradable diseño. 
Estos hoteles se pueden encontrar en áreas urbanas o rurales y en ellos siempre predomina el servicio personalizado y la intimidad dentro de sus instalaciones. Se llenan de arte y diseños novedosos, no tienen gran tamaño ni gran cantidad de personal, pero el equipo de trabajo que conforma su staff estará listo para realizar múltiples funciones y cubrir todas las necesidades del huésped que paga una tarifa alta por hospedarse en estos establecimientos.

\section{Desarrollo y Análisis de la Oferta}

El cantón Samborondón, dentro de la provincia del Guayas, se encuentra separado de Guayaquil por el río Daule, y es uno de los cantones con mayor desarrollo en los últimos años Municipio de Samborondón (2020). Dentro del cantón se encuentra el Parque Histórico Guayaquil, y dentro del parque está el Hotel del Parque con su restaurante llamado Casa Julián.

"El Parque Histórico Guayaquil es un programa cultural, medioambiental, educativo, recreativo y turístico que nos transporta en el tiempo hacia las costumbres y tradiciones de la antigua Provincia de Guayaquil, creada en 1.763, que comprendía casi la totalidad de la región costera del Ecuador. Cuenta con 8 hectáreas, divididas en tres zonas: la de Vida Silvestre, Zona de Tradiciones y Urbano Arquitectónica, las cuales conjugan lo natural, lo urbano y lo rural bajo un solo concepto: el rescate, conservación y promoción de nuestras tradiciones, constituyéndose en un lugar de recreación como medio de educación” Municipio de Samborondón (2020).

El Hotel cuenta con 44 habitaciones de lujo que armonizan perfectamente con la elegancia y la historia que posee su edificación. Ofrecen todos los servicios de un hotel de lujo, con la adición de encontrarse dentro de un atractivo turístico natural y cultural que le permite ser un oasis para los huéspedes que buscan tranquilidad con todos los servicios a su alcance.

Como lo indica en su estudio de campo Pallares (2019), el Hotel del Parque tiene las siguientes fortalezas:

Instalaciones de lujo arquitectónico, histórico-patrimonial, ya que se encuentra dentro de una edificación restaurada y preservada para poder mantener viva la historia de la ciudad, que sufrió varios incendios y perdió mucho del patrimonio arquitectónico.

Adicionalmente, como indica Baca Bonillo (2019), se intentó dejar una marca en su decoración, que demuestre la vida en la época republicana, por lo que existen áreas con estilo neoclásico como el Lounge que se encuentra en el piso alto del hotel. 
Amplias habitaciones: Las habitaciones del hotel no solo son lujosas y cálidas, también tienen aproximadamente 10 metros más que una habitación regular de un hotel del lujo, lo que las hace más atractivas a los ojos del huésped.

Los jardines: Los jardines poseen orquídeas, la flor de la ciudad de Guayaquil, tiene un jardín grande y huertos orgánicos, donde los huéspedes pueden deleitar su desayuno o inclusive el té de la tarde rodeado de una hermosa vegetación.

La ubicación y actividades: La ubicación privilegiada por encontrarse dentro del Parque histórico, le permite ofrecer actividades dentro de las áreas de naturaleza y vida silvestre, así como alrededor de sus plantaciones de cacao y banano. La sala de masaje que está ubicada en el antiguo campanario con una maravillosa vista al parque es uno de los servicios más exclusivos y diferentes que ofrece el hotel.

Servicios y experiencias: El hotel cuenta con muchos servicios de los que ya ofrecen los hoteles de lujo en la ciudad de Guayaquil; pero adicionalmente, ha creado las siguientes actividades o experiencias: Experiencia del café, Experiencia del Cacao, Huertos Orgánicos, Casa hacienda, Recorrido en lancha por el Rio, Charlas Históricas de Guayaquil, Atardecer con Vista a la ciudad y Búsqueda del tesoro (Niños) que son un elemento puntual y totalmente único dentro del sector en el que se encuentra.

Al revisar la oferta que tiene el hotel desde su infraestructura hasta sus servicios y paquetes de experiencia, se pueden establecer las razones por las que los huéspedes preferirían este hotel frente a los demás hoteles de lujo del gran Guayaquil.

\section{MATERIALES Y MÉTODOS}

El estudio es de tipo descriptivo pues analiza una realidad para poder describirla, asimismo cuenta con un enfoque cuantitativo, pues recurre a instrumentos de medición como son encuestas además de series de datos para hacer el diagnóstico del objeto de estudio.

El método científico utilizado es analítico-sintético puesto que va de lo general a lo particular y utiliza técnicas de investigación como la revisión documental, la revisión bibliográfica y la observación directa. Las fuentes son confiables y transparentes en su acceso y relacionadas con la hospitalidad y el turismo como lo son Trip Advisor, Booking y Expedia de amplio uso mundial.

Para determinar el tamaño de la muestra se utilizó la fórmula de poblaciones finitas lo que dio como resultado una muestra de 300 personas entre 25 y 54 años a quienes se encuestó de manera aleatoria dentro de la población adulta activa que se desplaza y se hospeda en hoteles de lujo en Guayaquil y Quito que son donde están ubicados los principales 
establecimientos de esta categoría, para determinar su conocimiento de los hoteles Boutique del país y de la ciudad, así como de la oferta específica del Hotel del Parque.

Una vez definida la metodología se procede a analizar los principales indicadores diagnóstico para luego ver los resultados de la encuesta.

\section{Ingreso de Turistas}

Para observar cuál es la participación e ingresos por países al Ecuador, y constatar de donde viene la mayoría de los turistas y potenciales huéspedes, el Ministerio de Turismo realiza un informe que indica el porcentaje de participación en llegadas, así como los millones de US\$ generados por país:

\begin{tabular}{|c|c|c|c|c|}
\hline & Miles de llegadas & Participación en llegadas & Variación anual de llegadas & Millones de US\$ generados \\
\hline 1 Colombia & 330,7 & $20,6 \%$ & $3,7 \%$ & 210,2 \\
\hline 2 Venezuela & 288,0 & $17,9 \%$ & $180,6 \%$ & 118,3 \\
\hline 3 Estados Unidos de América & 244,6 & $15,2 \%$ & $-8,4 \%$ & 399,1 \\
\hline 4 Perú & 139,1 & $8,6 \%$ & $-5,7 \%$ & 81,7 \\
\hline 5 España & 58,7 & $3,7 \%$ & $-6,8 \%$ & 96,4 \\
\hline 6 Argentina & 44,7 & $2,8 \%$ & $0,3 \%$ & 52,7 \\
\hline 7 Chile & 44,5 & $2,8 \%$ & $8,8 \%$ & 31,0 \\
\hline 8 Alemania & 33,4 & $2,1 \%$ & $8,3 \%$ & 38,2 \\
\hline 9 China & 30,1 & $1,9 \%$ & $33,4 \%$ & 28,7 \\
\hline 10 Canadá & 28,3 & $1,8 \%$ & $-5,2 \%$ & 27,9 \\
\hline 11 México & 27,5 & $1,7 \%$ & $2,3 \%$ & 17,0 \\
\hline 12 Reino Unido & 25,6 & $1,6 \%$ & $-11,4 \%$ & 30,9 \\
\hline 13 Francia & 25,4 & $1,6 \%$ & $10,0 \%$ & 37,4 \\
\hline 14 Cuba & 24,9 & $1,5 \%$ & $-4,4 \%$ & 15,8 \\
\hline 15 Brasil & 21,9 & $1,4 \%$ & $14,6 \%$ & 25,6 \\
\hline
\end{tabular}

Figura 1. Llegadas, participación e ingreso por países. Fuente: Ministerio de Turismo (2017).

La figura 1 indica que Colombia y Estados Unidos lideran los países con mayor llegada de turistas al país, con la excepción de Venezuela donde no se puede diferenciar a los turistas de los migrantes dadas las circunstancias actuales de ese país. Este análisis permite concluir que la mayoría de los huéspedes que se hospedan en el hotel son de Estados Unidos, primeramente.

En la tabla 1 que se presenta a continuación se podrá observar el análisis de oferta y demanda del grupo de hoteles de lujo de Guayaquil:

\section{Tabla 1}

Indicadores Hoteleros Completos 2018.

\begin{tabular}{|c|c|c|c|c|c|c|c|c|c|}
\hline \multicolumn{10}{|c|}{ ANALISIS DE LA OFERTA Y LA DEMANDA } \\
\hline & HAB & $\begin{array}{l}\text { HAB. } \\
\text { DISP. }\end{array}$ & $\begin{array}{c}\text { \% PART } \\
\text { MDO }\end{array}$ & $\%$ OCUP. & $\begin{array}{l}\text { HAB. } \\
\text { OCUP }\end{array}$ & $\begin{array}{c}\text { \% PART } \\
\text { REAL }\end{array}$ & ADR & VENTA & $\begin{array}{l}\text { REV } \\
\text { PAR }\end{array}$ \\
\hline Oro Verde & 236 & 28.320 & $12,00 \%$ & $59,46 \%$ & 16.838 & $11,29 \%$ & $\$ 80,71$ & $\$ 1.359 .025$ & $\$ 47,99$ \\
\hline $\begin{array}{l}\text { Hotel del } \\
\text { Parque }\end{array}$ & 44 & 5.280 & $2,24 \%$ & $44,51 \%$ & 2.350 & $1,58 \%$ & $\$ 237,28$ & $\$ 557.652$ & $\$ 105,62$ \\
\hline
\end{tabular}




\begin{tabular}{lccccccccc}
\hline Unipark & 140 & 16.800 & $7,12 \%$ & $65,92 \%$ & 11.075 & $7,43 \%$ & $\$ 67,14$ & $\$ 743.557$ & $\$ 44,26$ \\
Hilton & 294 & 35.280 & $14,95 \%$ & $59,78 \%$ & 21.091 & $14,14 \%$ & $\$ 114,10$ & $\$ 2.406 .422$ & $\$ 68,21$ \\
Wyndham & 175 & 21.000 & $8,90 \%$ & $69,87 \%$ & 14.673 & $9,84 \%$ & $\$ 113,10$ & $\$ 1.659 .486$ & $\$ 79,02$ \\
Sheraton & 141 & 16.920 & $7,17 \%$ & $71,18 \%$ & 12.043 & $8,08 \%$ & $\$ 112,99$ & $\$ 1.360 .824$ & $\$ 80,43$ \\
Holiday Inn & 122 & 14.640 & $6,20 \%$ & $68,99 \%$ & 10.100 & $6,77 \%$ & $\$ 105,50$ & $\$ 1.065 .507$ & $\$ 72,78$ \\
Radisson & 85 & 10.200 & $4,32 \%$ & $77,66 \%$ & 7.922 & $5,31 \%$ & $\$ 81,96$ & $\$ 649.269$ & $\$ 63,65$ \\
Sonesta & 112 & 13.440 & $5,69 \%$ & $69,39 \%$ & 9.326 & $6,25 \%$ & $\$ 100,46$ & $\$ 936.942$ & $\$ 69,71$ \\
Courtyard & 144 & 17.280 & $7,32 \%$ & $68,26 \%$ & 11.795 & $7,91 \%$ & $\$ 101,24$ & $\$ 1.194 .111$ & $\$ 69,10$ \\
Wyndham & 108 & 12.960 & $5,49 \%$ & $76,44 \%$ & 9.907 & $6,64 \%$ & $\$ 82,63$ & $\$ 818.626$ & $\$ 63,17$ \\
Garden & 182 & 21.840 & $9,25 \%$ & $40,65 \%$ & 8.877 & $5,95 \%$ & $\$ 75,32$ & $\$ 668.664$ & $\$ 30,62$ \\
Guayaquil & 196 & 10.680 & $4,52 \%$ & $65,12 \%$ & 6.955 & $4,66 \%$ & $\$ 64,59$ & $\$ 449.238$ & $\$ 42,06$
\end{tabular}

Fuente: Gerencia (2019).

El análisis de esta tabla 1 permite establecer un comparativo en REV PAR (Revenue per available room) o ingreso por habitación disponible, y así constatar si la tarifa sería un impedimento en la llegada de huéspedes al hotel. El buen performance del hotel permite ser el único hotel tipo boutique que compite en esta categoría.

El Revpar sobrepasa la tarifa promedio de la ciudad, ya que sus huéspedes son exclusivos y generalmente por su tamaño y categoría no recibe grupos grandes que implican bajas en sus tarifas.

En la figura 2 a continuación, en las estadísticas del Ministerio de Turismo se observa la tasa de ocupación y tarifa promedio por habitación por destino para hoteles 5 estrellas: 


\section{COMPARATIVO POR DESTINOS}

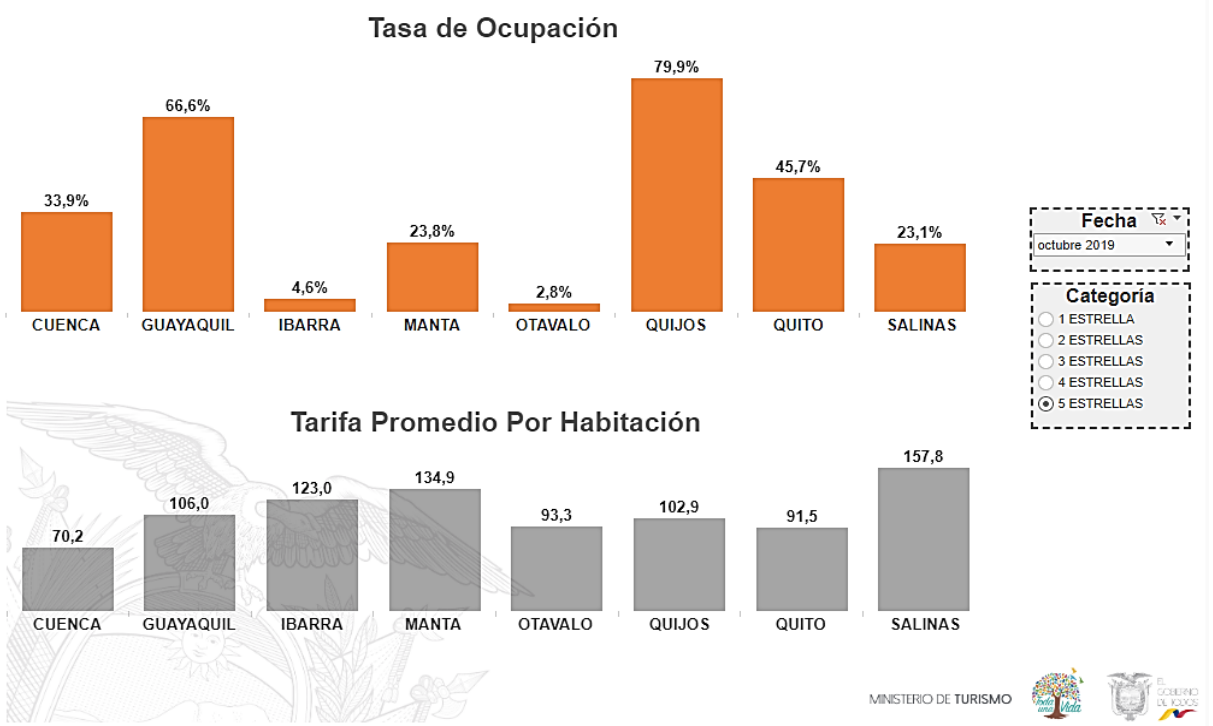

Figura 2. Indicadores Coyunturales Alojamiento. Fuente: Ministerio de Turismo (2019).

La figura 2 analiza el promedio de ocupación y tarifa del país por destino, y aún en este comparativo macro se puede observar que el hotel se encuentra sobre la media en cuanto a su tarifa, y muy cerca de la media en \% de ocupación confirmando que sus números son competitivos siendo el único hotel boutique de lujo compitiendo con hoteles de marca mucho más grandes ubicados dentro de la ciudad.

El Hotel del Parque recibe principalmente al mercado Tour \& Travel, que es el que se hospeda en Guayaquil una noche antes de partir hacia Galápagos principalmente. Otro de los mercados es el diplomático que suele venir por reuniones puntuales a Guayaquil y Quito, y escoge el hotel como sitio de descanso y de reuniones privadas y pequeñas.

El mercado de bodas en Guayaquil ha incrementado la ocupación del hotel, ya que los novios prefieren pasar su noche de bodas en uno de los mejores hoteles de la ciudad, lejos del ruido de la ciudad.

\section{RESULTADOS}

En el resumen de las preguntas más relevantes se consulta si conocen los hoteles boutique de la ciudad, y del país, y su preferencia por alguno en específico.

También sobre la preferencia en los servicios que puede ofrecer un hotel, y el conocimiento de los servicios específicos del hotel del Parque.

Conocimiento de hoteles boutique en Ecuador basado en una selección aleatoria de hoteles en las principales ciudades del país: 


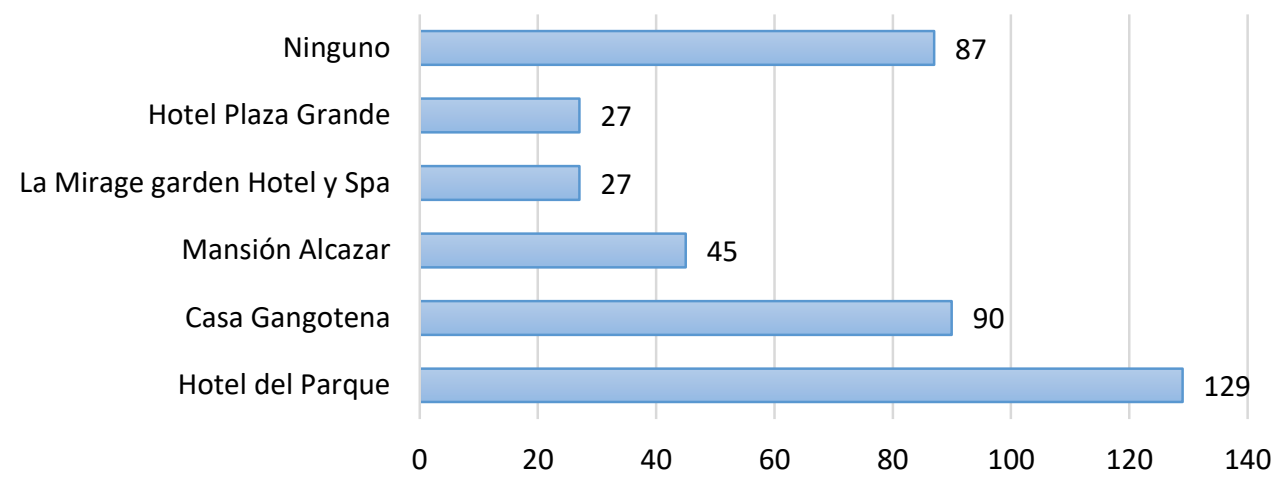

Figura 3. Indicadores Coyunturales Alojamiento. Fuente: Elaboración propia.

Como se puede observar en la figura 3 de los 300 encuestados, 129 personas que equivalen al $43 \%$ han escuchado de Hotel del Parque como uno de los hoteles boutique del país, inclusive se ha posicionado como el más conocido dentro de la lista a elegir. Pero también se puede observar que el siguiente valor que es 87 equivalente al $29 \%$, indica el número de personas que no conoce ninguno de los hoteles boutique mencionados.

Conocimiento de hoteles boutique en Guayaquil, basado en la selección de hoteles boutique de la ciudad:

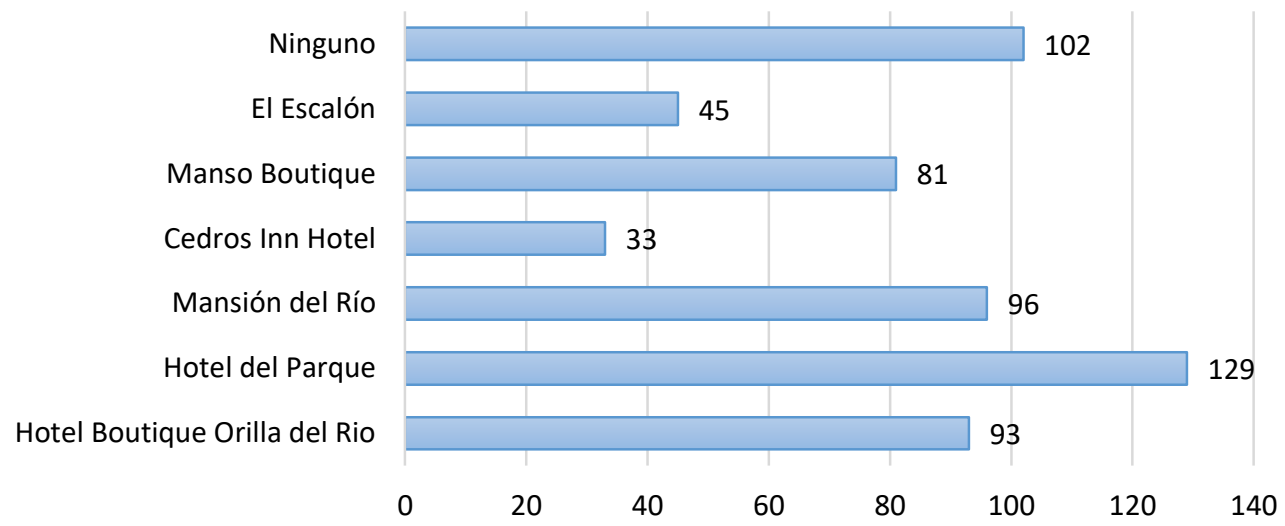

Figura 4. Conocimiento de hoteles boutique en Guayaquil. Fuente: Elaboración propia.

Como se puede observar en la figura 4, 129 personas que equivalen al $43 \%$ han escuchado de Hotel del Parque como uno de los hoteles boutique de Guayaquil, y también dentro de la ciudad se posiciona en primer lugar. Nuevamente se puede observar que el siguiente valor (102) equivale a los que desconocen por completo la existencia de hoteles boutique en la ciudad. Número de noches de estadía en la ciudad de Guayaquil: 


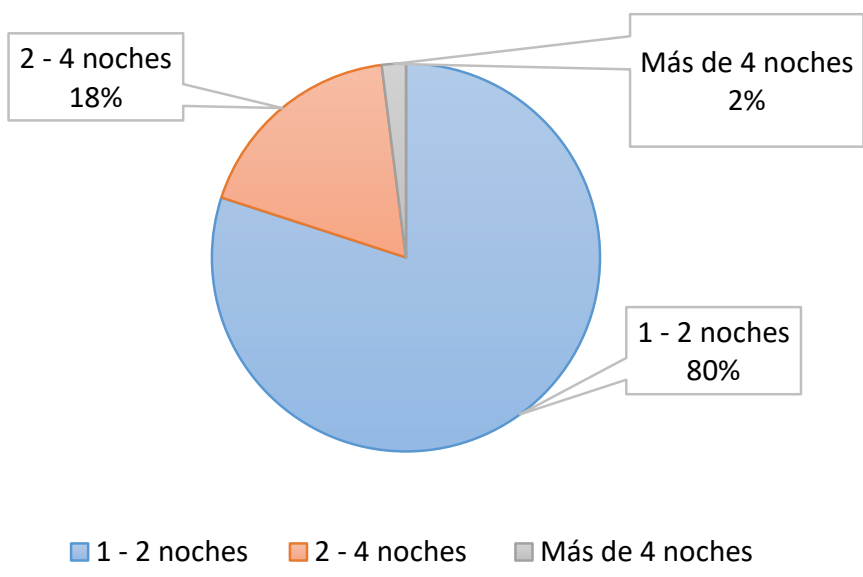

Figura 5. Número de noches de hospedaje en Guayaquil. Fuente: Elaboración propia.

En la figura 5 se observa que la ciudad de Guayaquil es considerada como un destino de paso, donde los huéspedes generalmente se hospedan para llegar luego a su destino final en el caso de Tour and Travel; en el caso del cliente corporativo, se hospeda pocas noches ya que viene por razones puntuales y no requiere mucho tiempo en la ciudad. El $80 \%$ de los encuestados indicaron que se hospedan solo de 1 a dos noches. Motivo de viaje a la ciudad de Guayaquil:

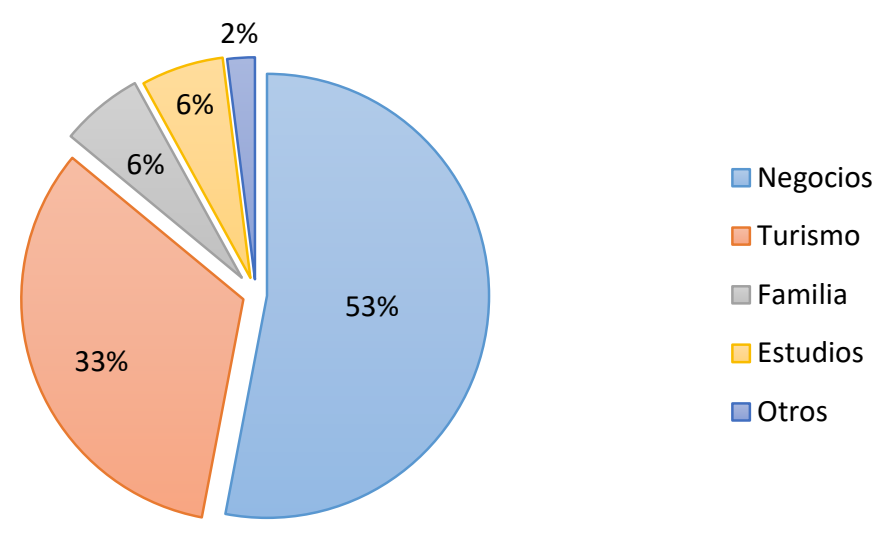

Figura 6. Motivo de viaje en hotel de lujo a la ciudad de Guayaquil. Fuente: Elaboración propia.

En la figura 6 el 53\% confirma que sus viajes a la ciudad son por negocios, esto incluye participación en congresos y convenciones, y un 33\% viaja por Turismo generalmente con destino final en las Islas Galápagos; lo que indica que Guayaquil todavía no se considera un destino final para descanso y ocio. Preferencia de oferta de servicios adicionales dentro del hotel: 


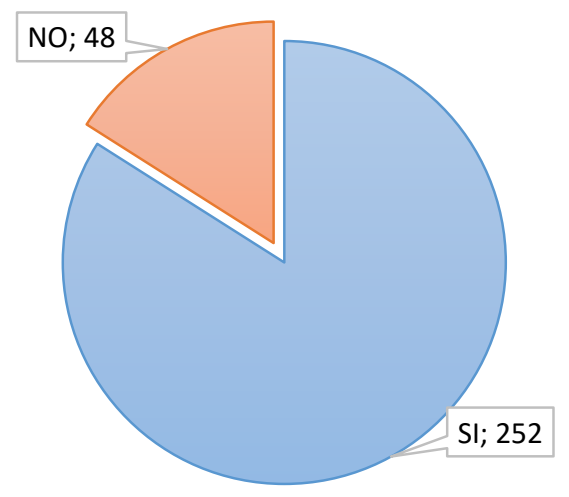

Figura 7. Preferencia del huésped en oferta de servicios adicionales. Fuente: Elaboración propia.

Como se puede observar en la figura 7, los huéspedes que se hospedan en hoteles de lujo si prefieren tener una oferta de servicios adicionales dentro de las instalaciones del hotel. En este caso es un equivalente al $84 \%$. De esta manera pueden acceder con más rapidez, y mantener un servicio exclusivo y personalizado ya que es otorgado por el mismo prestador del servicio de hospedaje. Conocimiento de los servicios de experiencias del Hotel del Parque:

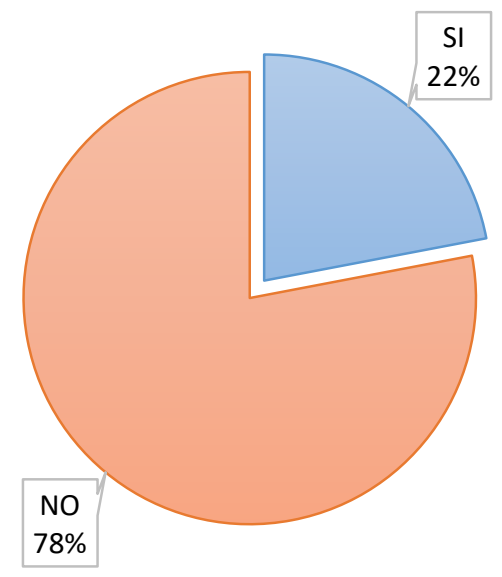

Figura 8. Conocimiento de la oferta de servicios del Hotel del Parque. Fuente: Elaboración propia.

De acuerdo a la figura 8 , El 78\% de los encuestados no conocían la oferta que el hotel tiene, tanto en servicios dentro del hotel como en actividades y experiencias, lo que concluye en una falta de publicidad para promocionarse con su oferta completa. Del $22 \%$ que conocía de las ofertas, un grupo ya se ha hospedado anteriormente en el hotel, y otros tienen negocios o conocimiento más cercano del hotel y del parque histórico Guayaquil. 


\section{Análisis de Percepción del Cliente en las OTAS}

En función de la figura 9, dentro de la percepción de la experiencia del huésped para Expedia, se califican la limpieza, el servicio del personal, los amenities o artículos que se incluyen en la tarifa, así como las condiciones del hotel. En los 5 parámetros que se revisan, en esta página el $100 \%$ de los huéspedes lo ha calificado como excepcional.

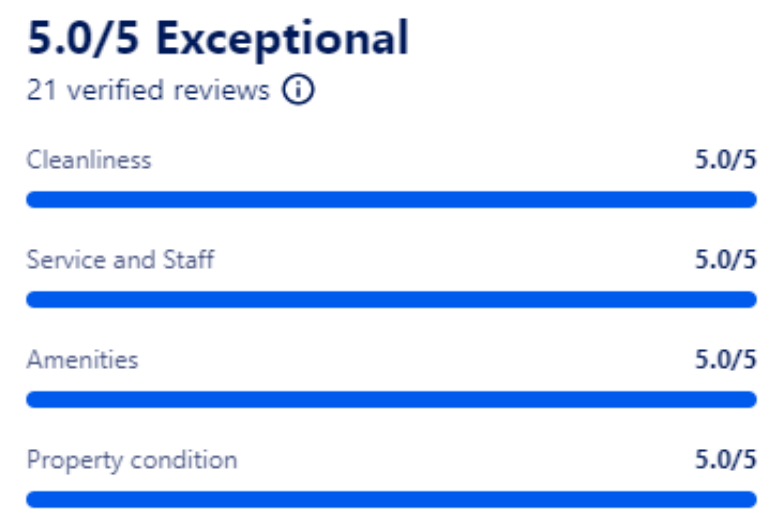

Figura 9. Percepción del Hotel del Parque \#1. Fuente: Expedia (2021).

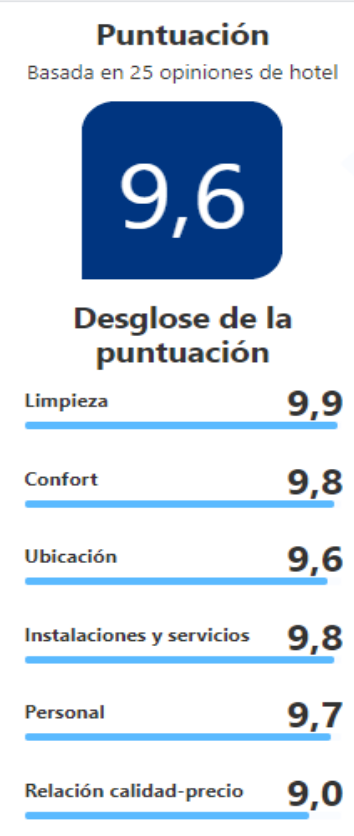

Figura 10. Percepción del Hotel del Parque \#2. Fuente: Booking (2021).

Dentro de la percepción de la experiencia del huésped para booking.com, se califican la limpieza, la ubicación, las instalaciones y servicio, el personal, y la relación calidad-precio. En los 6 parámetros que se revisan en esta página, el puntaje medio que han otorgado es de 9.6/10 lo que también le da una excelente puntuación. 


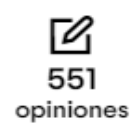

\section{Opiniones}

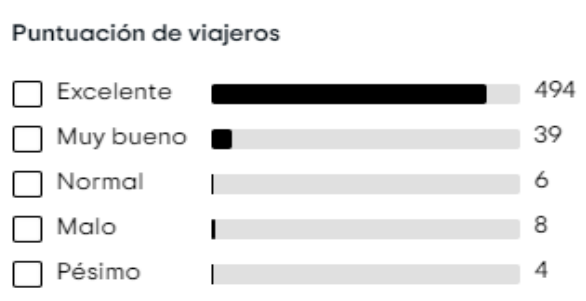

Figura 11. Percepción del Hotel del Parque \#3. Fuente: Tripadvisor (2021).

Según lo observado en la Figura 11, Dentro de la percepción de la experiencia del huésped para Tripadvisor, se califican las opiniones de las experiencias de los viajeros como excelente, muy bueno, normal, malo y pésimo. En los 5 parámetros que se revisan en esta página, el $89.66 \%$ de los viajeros lo califican como excelente, vs el $0.73 \%$ que lo califica como pésimo.

Luego de revisar, analizar y comparar los diferentes comentarios emitidos por huéspedes de tres de las más importantes agencias de viajes virtuales que llegan al hotel, se puede confirmar que tanto las instalaciones como el excelente servicio que se ofrece, posicionan al hotel como uno de los mejores de Guayaquil.

Una vez que el huésped hace contacto con el hotel, los diferentes departamentos se encargan de ofrecer toda la gama de servicios que tienen, así como de preguntar constantemente a sus huéspedes qué necesitan para que su estadía sea mejor.

\section{CONCLUSIONES}

Al determinar cómo se percibe el servicio en el Hotel del Parque dentro de la oferta de hoteles de lujo en Guayaquil y luego de analizar las bases teóricas y los datos estadísticos de cómo la hotelería de lujo, específicamente el tipo de hotel boutique poco a poco está ingresando al mercado de hoteles de Guayaquil y del Ecuador, se concluye que este tipo de establecimientos ha encontrado un nicho de mercado importante dentro de una ciudad que principalmente se considera de paso o de negocios.

En el caso específico del hotel del Parque, se ha constatado que ya se encuentra posicionado como parte de los hoteles de lujo y de excelencia de la ciudad, donde su servicio de primera concuerda con la relación calidad-precio que el huésped de este tipo de hotel está buscando. 
Una vez que el hotel se encuentra en el radar del futuro huésped, la oferta de servicios de calidad que tienen, más sus atributos únicos por su ubicación y edificación, reafirman que el hospedarse en el hotel permite experimentar la ciudad de una manera mucho más refinada y a la vez cubriendo el ciclo de la experiencia con gran satisfacción para quien lo consume.

El análisis final refleja que el principal mercado del hotel es el extranjero, por lo que los aliados estratégicos por donde se vende el hotel si ofrecen los servicios y experiencias que posee actualmente, pero que también deberían ser reconocidas por el mercado nacional que está viajando y consumiendo noches hoteleras, y de esta manera subir aún más la posición del hotel aportando en un incremento de ocupación y tarifa promedio ya que no tiene mayor competencia dentro de la ciudad.

\section{REFERENCIAS BIBLIOGRÁFICAS}

Acosta, A., Fernandez, N., \& Mollón, M. (2002). Recursos humanos en empresas de turismo y hostelería. Madrid: Pearson Education S.A.

Administración del Señor Lcdo. Lenin Moreno Garcés . (2018). Derecho Ecuador. Obtenido de https://derechoecuador.com/registro-oficial/2018/11/registro-oficial-no365-lunes12-de-noviembre-de-2018

Baca Bonillo, F. (2019). Trabajo de campo: Entrevista al Gerente General del Hotel Parque. (J. Pallares, Entrevistador)

Beeho, A., \& Prentice, R. (1997). Conceptualizing the experiences of heritage tourists: A case study of New Lanark World Heritage Village. Tourism Management, 75-87.

Booking Reseñas y Valoraciones. (28 de agosto de 2020). Booking.com. Obtenido de Booking.com: https://www.booking.com/hotel/ec/del-parque-guayaquil.es.html

Cisneros Garrido, G. (2015). Posicionamiento estratégico y gestión de marcas turísticas. Obtenido de Papers de Turisme: http://www.papersdeturisme.gva.es/ojs/index.php/Papers/article/viewFile/315/269

Contardo, N., \& Wenger, G. (2012). Hotel Boutique en Casa Cingolani. Obtenido de INSTITUTO SUPERIOR NOL": https://repotur.yvera.tur.ar/bitstream/handle/123456789/4793/WENGER\%20Y\%20C ONTARDO.\%20TESINA\%20REPOTUR.pdf?sequence=1\&isAllowed=y

Díaz-Bustamante, M. (2013). Metodología para la investigación del posicionamiento. En ACTITUDES Y PERCEPCIONES SOBRE LAS FRAGANCIAS. Madrid: 38-50. 
Empresa Pública Municipal de Turismo, Promoción Cívica y Relaciones Internacionales de Guayaquil, EP. . (2019). Guayaquil es mi Destino . Obtenido de http://www.guayaquilesmidestino.com/es/naturaleza/afuera-de-guayaquil/parquehistorico

Gerencia. (2019). Entrevista Gerencia Hotel del Parque Histórico. (J. Pallares, Entrevistador) Hoseltur LATAM. (2015). OMT identifica criterios para la clasificación de hoteles de 4 y 5 estrellas. Obtenido de Hosteltur Latam: https://www.hosteltur.com/lat/125000_omtidentifica-criterios-clasificacion-hoteles-4-5-estrellas.html

Hotel del Parque Histórico. (28 de agosto de 2020). hoteldelparquehistorico.com. Obtenido de hoteldelparquehistorico.com: https://www.hoteldelparquehistorico.com/es/

Hotel del Parque Histórico Guayaquil. (2019). Hotel del Parque . Obtenido de Hotel del Parque Histórico: https://www.hoteldelparquehistorico.com/es/nuestro-hotel.htm

Ibáñez, J., \& Manzano, R. (2007). Posicionamiento: Elemento clave de la estraegia de Marketing. Obtenido de IE Business school: http://accioneduca.org/admin/archivos/clases/material/posicionamiento_1563557948. pdf

Kotler, P., \& Bowen T., J. (2015). Fundamentos de Marketing (8va Edición ed.). Naucalpan, Estado de México, México: PEARSON EDUCACIÓN.

Ministerio de Turismo. (2016). Reglamiento de alojamiento turístico. Obtenido de Ministerio de turismo: https://www.turismo.gob.ec/wp-content/uploads/2016/06/REGLAMENTODE-ALOJAMIENTO-TURISTICO.pdf

Ministerio de Turismo. (2017). Turismo en Cifras. Obtenido de https://servicios.turismo.gob.ec/descargas/Turismocifras/Publicaciones/Perfiles/PerfilesDeTurismolnternacional.pdf

Ministerio de Turismo. (25 de septiembre de 2018). Indicadores Coyunturales de Alojamiento. Obtenido de Sitio web oficial: http://servicios.turismo.gob.ec/index.php/turismocifras/2018-09-18-21-11-17/indicadores-de-alojamiento

Ministerio de Turismo. (2016). ACUERDO No. 20150102. MANUAL DE APLICACIÓN DEL REGLAMENTO DE ALOJAMIENTO TURÍSTICO. . Quito: Registro Oficial.

Morgan, M., Elbe, J., \& Curiel, J. (2019). Has the Experience Economy Arrived? The Views of Destination Managers in Three Visitor-dependent Areas. INTERNATIONAL JOURNAL OF TOURISM RESEARCH, 16. 
Municipio de Samborondón. (2020). Samborondón - Historia. Obtenido de Municipio de Samborondón: https://www.samborondon.gob.ec/actividad-economica-y-productiva/

Pallares, J. (2019). "Estrategias para el posicionamiento y la comercialización efectiva de hoteles de lujo. Caso: Hotel del Parque, Ecuador". Trabajo de titulación. Ecuador: Universidad Ecotec.

Pine II, J., \& Gilmore, J. (1998). Welcome to the Experience Economy. Obtenido de Harvard Business Review Home: https://hbr.org/1998/07/welcome-to-the-experience-economy

Revista Empresarial \& Laboral. (2018). RevistaEmpresarial.com. Obtenido de RevistaEmpresarial.com: $\quad$ https://revistaempresarial.com/turismo/destinos/lahoteleria-de-lujo-ofrece-experiencias-unicas-a-sus-huespedes/

Rey, J., Zambrano, M., \& Zambrano, P. (2015). Comunicación y márketing: ¿convivencia o integración? QUESTIONES PUBLICITARIAS, 117-127.

Sánchez Aguirre, D., Maldonado Alcudia, M., Martínez Vázquez, L., \& Salinas Villareal, V. (2017). Experiencia de usuarios en hoteles. Obtenido de The Institute for Business and Finance Research: file:///C:/Users/Alejandra\%20Ycaza/Downloads/SSRNid2827881\%20(2).pdf

UNWTO. (2015). Hotel Classification Systems: Recurrence of criteria in 4 and 5 star hotels. Obtenido de World Tourism Organization: https://www.eunwto.org/doi/pdf/10.18111/9789284416646

Weiermair, K. (Enero de 1997). Calidad de servicios y su gestión en las empresas turísticas. Obtenido de Research

Gate: https://www.researchgate.net/publication/312786709_Calidad_de_servicios_y_su_ge stion_en_las_empresas_turisticas

Ycaza, A. (2019). Análisis de la importancia del departamento de ventas para un hotel nuevo caso Hotel en el cantón Durán. Obtenido de Universidad Ecotec: https://ecotec.edu.ec/content/uploads/2019/12/memorias20192.pdf 